\title{
EELS and EFTEM Imaging of Surface Plasmons in Silica-Ag Core-Shell Nanoparticles.
}

\author{
A. S. Eggeman, * A. K. Petford-Long,** P. J. Dobson*** \\ * Dept of Materials, University of Oxford, Oxford, OX1 3PH, UK \\ **Materials Science Division, Argonne National Lab, 9700 S Cass Ave, Argonne, IL 60439 \\ ***Begbroke Science Park, University of Oxford, Oxford, OX5 1PF, UK
}

Nanoparticles of $\mathrm{Ag}$ and $\mathrm{Au}$ are of interest because they exhibit a surface plasmon resonance at visible frequencies, with the wavelength of the plasmon being variable as a function of particle size.(1) However the range of frequencies over which the plasmon can be tuned is relatively small. If however the metal is in the form of a shell surrounding a dielectric core, the resonant condition is determined by the ratio of the core diameter to the shell thickness, giving a much greater flexibility in tuning the resonant frequency across the visible spectrum.(2) The most widely used technique to detect surface plasmon resonances is ultraviolet-visible (UV-Vis) spectroscopy however this can only look at the signal averaged over a large number of particles (of the order of $1 \times 10^{12}$ ).

We have used electron energy loss spectroscopy (EELS) to record the surface plasmon peak from single silica-Ag core-shell nanoparticles and energy-filtered TEM (EFTEM) to visualize the spatial extent of the surface plasmon. $100 \mathrm{~nm}$ diameter silica spheres were produced using the aqueous chemistry Stöber process.(3) The surface of the cores was seeded with Au nanoparticles and a silver shell was attached to each core by dispersing the cores in ammoniacal silver nitrate solution and reducing using formaldehyde. The shell thickness could be varied by altering the volume of silver nitrate solution. The EELS and EFTEM data were recorded using a JEOL 3000F FEGTEM fitted with a Gatan imaging filter (GIF).

The low-loss region of an EELS spectrum recorded from a single core-shell particle with a $22 \mathrm{~nm}$ thick Ag shell is shown in Fig. 1. The spectrum was recorded with a selected area aperture inserted, and the zero loss peak has been subtracted. There is a large peak at an energy loss of $2.6 \mathrm{eV}$, Extending from energy loss peak at $2.6 \mathrm{eV}$ is a shoulder at an energy of approximately 7-8 eV and finally a broad bulk plasmon extending from $12 \mathrm{eV}$ to over $30 \mathrm{eV}$. An energy loss of $2.6 \mathrm{eV}$ corresponds well to a wavelength of $540 \mathrm{~nm}$, at which a surface plasmon resonance peak was observed in UV-Vis spectra recorded from larger volumes of the same particles. Figure 2 includes a series of EFTEM images recorded at energy losses of (a) $0 \mathrm{eV}$, (b) $3 \mathrm{eV}$, (c) $7 \mathrm{eV}$, and (d) $14 \mathrm{eV}$. The images were recorded using a slit width of $2 \mathrm{eV}$. The dark Ag shell is clearly visible in Fig. 2(a), surrounding a lighter silica shell. The Ag shell is not single crystal but is composed of many crystallites that have coalesced to form a continuous shell. The $3 \mathrm{eV}$ energy loss image (Fig. 2(b)) shows a bright halo surrounding the Ag shell and extending out from the particle surface, confirming that the peak at $2.6 \mathrm{eV}$ does correspond to the surface plasmon resonance of the Ag shell. As the energy loss is increased to $7 \mathrm{eV}$ (Fig 2(c)) so the halo becomes confined to the shell surface and for an energy loss of $14 \mathrm{eV}$ (Fig. 2(d)) the contrast in the entire image is brighter, in addition to the surface halo, indicating that the high energy-loss peak results from a bulk plasmon with contributions from the core, shell and substrate.

1. G. Mie, Ann. Phys (Leipzig) 24, 377 (1908).

2. R. D. Averitt, S. L. Westcott, and N. J. Halas, Journal of the Optical Society of America B 16, 1824 (1999).

3. W. Stoeber, A. Fink, and E. Bohn, Journal of Colloid and Interface Science 26, 62 (1968). 


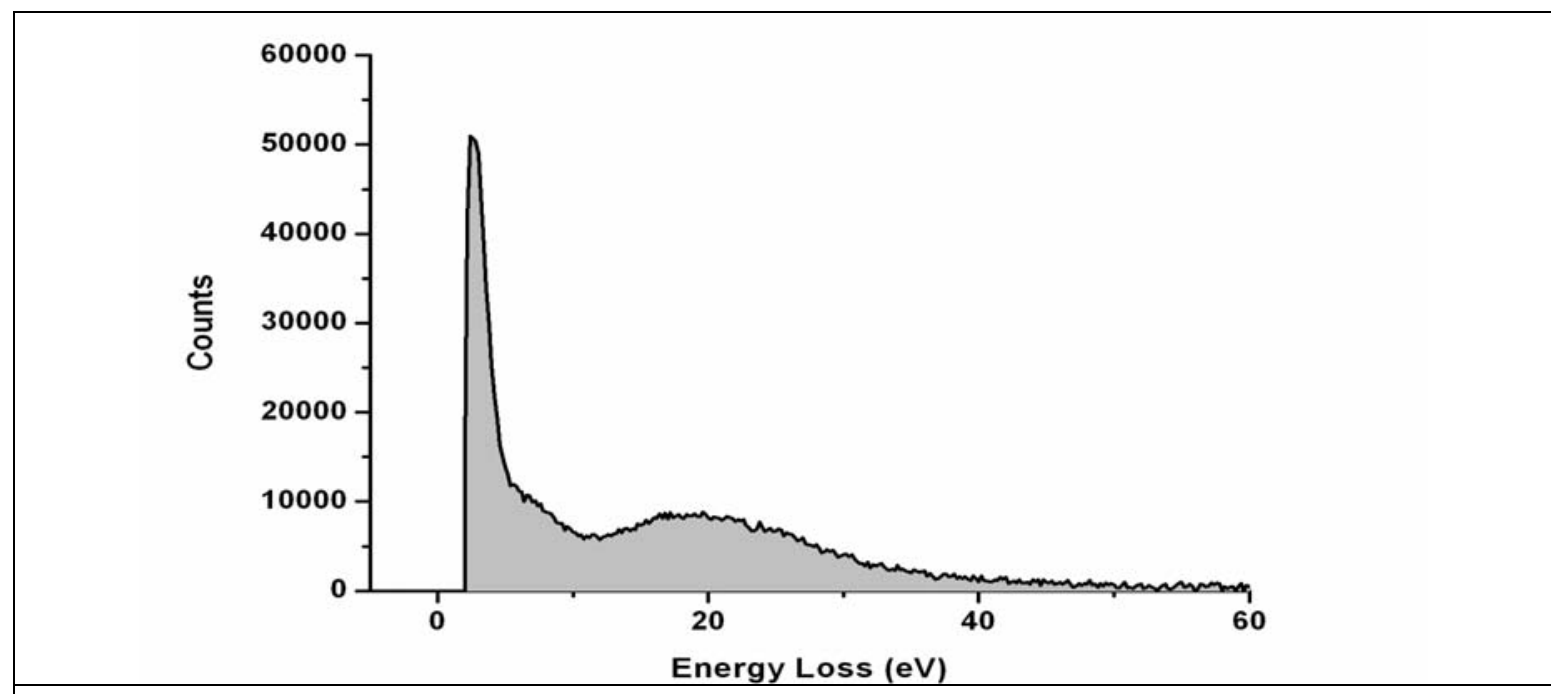

Figure 1. EELS absorption spectrum from a $100 \mathrm{~nm}$ silica core coated with a $22 \mathrm{~nm}$ silver shell, after removal of the zero-loss peak.

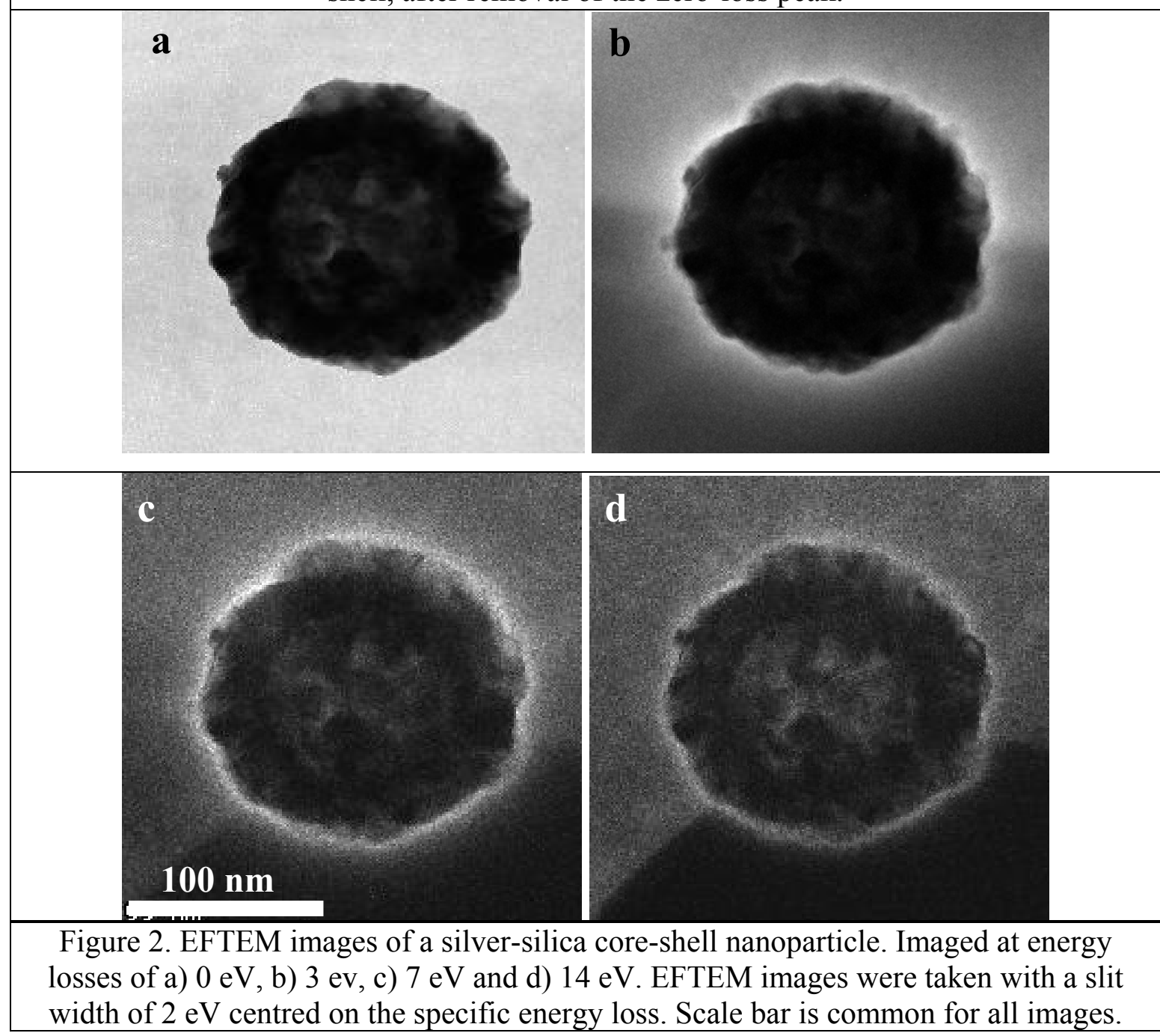

